\title{
A CLASS OF MULTILINEAR OSCILLATORY SINGULAR INTEGRALS RELATED TO BLOCK SPACES
}

\author{
SHANZHEN LU AND HUOXIONG Wu
}

(Received January 15, 2002, revised March 23, 2004)

\begin{abstract}
This paper is devoted to the study on the $L^{p}$-mapping properties for a class of multilinear oscillatory singular integrals with polynomial phase and rough kernel. By means of the method of block decomposition for the kernel function, the authors show that for any non-trivial polynomial phase, the $L^{p}\left(\boldsymbol{R}^{n}\right)$ boundedness of the multilinear oscillatory singular integral operators and that of the corresponding local multilinear singular integral operators are equivalent; and for any real-valued polynomial phase, the $L^{p}\left(\boldsymbol{R}^{n}\right)$ boundedness of the multilinear oscillatory integral operators can be deduced from that of the corresponding multilinear singular integral operators.
\end{abstract}

1. Introduction and main results. It is well known that the oscillatory singular integral with polynomial phase has arisen in the study of Hilbert transforms along curves, singular integrals supported on lower-dimensional varieties and singular Radon transforms etc. Since Ricci and Stein [19] established a celebrated result to the effect that a class of oscillatory singular integrals with polynomial phase and smooth kernel is bounded on $L^{p}\left(\boldsymbol{R}^{n}\right)(1<p<\infty)$, there has been significant progress in the study of these operators (see [7], [10], [16], [17] etc.). In particular, Lu and Zhang [17] extended the above result of [19] to the rough kernel case and found out a simple criterion for $L^{p}$-boundedness of these operators. Recently, the authors [16], by the method of block decomposition for the kernel function, also gave an essential improvement of the result of [17]. Meanwhile, we obtained a criterion for the weighted $L^{p}$-boundedness for higher order commutators of this kind of oscillatory operators.

In this paper, we will consider the following multilinear oscillatory singular integral operator $T^{A}$ defined by

$$
T^{A} f(x)=\text { p.v. } \int_{\boldsymbol{R}^{n}} e^{i P(x, y)} \frac{\Omega(x-y)}{|x-y|^{n+m}} R_{m+1}(A ; x, y) f(y) d y, \quad n \geq 2,
$$

where $P(x, y)$ is a real polynomial on $\boldsymbol{R}^{n} \times \boldsymbol{R}^{n}, \Omega$ is homogeneous of degree zero on $\boldsymbol{R}^{n}$, $R_{m+1}(A ; x, y)$ denotes the $(m+1)$-st $(m \geq 1)$ remainder of the Taylor series of $A$ at $x$ about $y$, more precisely,

$$
R_{m+1}(A ; x, y)=A(x)-\sum_{|\gamma| \leq m} \frac{1}{\gamma !} D^{\gamma} A(y)(x-y)^{\gamma}
$$

2000 Mathematics Subject Classification. Primary 42B20; Secondary 42B25.

Research was supported by the National 973 Project (G19990751) and SEDF of China. 
and $D^{\gamma} A \in \operatorname{BMO}\left(\boldsymbol{R}^{n}\right)$ for all multi-indices $|\gamma|=m$.

In 1998, Chen, Hu and $\mathrm{Lu}$ [3] gave a criterion for the $L^{p}\left(\boldsymbol{R}^{n}\right)$ boundedness of $T^{A}$ for any nontrivial real-valued polynomial $P(x, y)$ and $\Omega \in \cup_{r>1} L^{r}\left(S^{n-1}\right)$. Afterwards, Ding and $\mathrm{Lu}$ [8] extended the above result to the weighted case. Recently, Chu, $\mathrm{Hu}$ and $\mathrm{Lu}$ [4] improved the result of [3] to the case $\Omega \in L\left(\log ^{+} L\right)^{2}\left(S^{n-1}\right)$.

On the other hand, for the block spaces $B_{q}^{0, v}\left(S^{n-1}\right)(q>1, v=0,1)$ introduced by Jiang and Lu (see [15]), Keitoku and Sato [12] pointed out that

$$
\bigcup_{r>1} L^{r}\left(\boldsymbol{R}^{n}\right) \subset B_{q}^{0,1}\left(S^{n-1}\right) \subset B_{q}^{0,0}\left(S^{n-1}\right),
$$

which are proper inclusions. It is easy to see from [12] that $B_{q}^{0,0}\left(S^{n-1}\right)$ is not contained in $L\left(\log ^{+} L\right)^{2}\left(S^{n-1}\right)$ although the relationship between $B_{q}^{0,0}\left(S^{n-1}\right)$ (or $\left.B_{q}^{0,1}\left(S^{n-1}\right)\right)$ and $L \log ^{+} L\left(S^{n-1}\right)$ remains open. It is natural to ask whether the condition on $\Omega$ in [3] can be weakened to the case of $\Omega \in B_{q}^{0,0}\left(S^{n-1}\right)$ or $\Omega \in B_{q}^{0,1}\left(S^{n-1}\right)$. The main purpose of this paper is to give a positive answer to the above question. Before stating our results, let us first review some concepts.

Definition 1 (cf. [17]). (i) A real-valued polynomial $P(x, y)$ is said to be non-trivial if $P(x, y)$ cannot be written as $P_{0}(x)+P_{1}(y)$, where $P_{0}$ and $P_{1}$ are both polynomials defined on $\boldsymbol{R}^{n}$.

(ii) A non-trivial polynomial $P(x, y)$ is said to have the property $\mathcal{P}$, if $P(x, y)$ satisfies

$$
P(x, y)=P(x-h, y-h)+R_{0}(x, h)+R_{1}(y, h), h \in \boldsymbol{R}^{n},
$$

where $R_{0}$ and $R_{1}$ are both real polynomials defined on $\boldsymbol{R}^{n} \times \boldsymbol{R}^{n}$.

(iii) A non-trivial polynomial $P(x, y)$ is said to be non-degenerate, if for positive integers $k$ and $l$,

$$
P(x, y)=\sum_{|\alpha| \leq k,|\beta| \leq l} a_{\alpha \beta} x^{\alpha} y^{\beta} \text { and } \sum_{|\alpha|=k,|\beta|=l}\left|a_{\alpha \beta}\right|>0 .
$$

Definition 2 (cf. [15]). A $q$-block on $S^{n-1}$ is an $L^{q}(1<q \leq \infty)$ function $b(\cdot)$ that satisfies

$$
\text { (i) } \operatorname{supp}(b) \subseteq Q, \quad \text { (ii) }\|b\|_{L^{q}\left(S^{n-1}\right)} \leq|Q|^{1 / q-1} \text {, }
$$

where $Q=S^{n-1} \cap\left\{y \in \boldsymbol{R}^{n}:|y-\varsigma|<\rho\right.$ for some $\varsigma \in S^{n-1}$ and $\left.\rho \in(0,1]\right\}$.

DEFINITION 3 (cf. [15]). For $v \geq 0$, the block spaces $B_{q}^{0, v}$ on $S^{n-1}$ are defined by

$$
B_{q}^{0, v}\left(S^{n-1}\right)=\left\{\Omega \in L^{1}\left(S^{n-1}\right): \Omega\left(y^{\prime}\right)=\sum_{s} C_{s} b_{s}\left(y^{\prime}\right), M_{q}^{0, v}\left(\left\{C_{s}\right\}\right)<\infty\right\},
$$

where each $C_{S}$ is a complex number, each $b_{s}$ is a $q$-block supported in $Q_{s}$, and

$$
M_{q}^{0, v}\left(\left\{C_{s}\right\}\right)=\sum_{s}\left|C_{s}\right|\left\{1+\left(\log ^{+} \frac{1}{\left|Q_{s}\right|}\right)^{v+1}\right\} .
$$


It should be pointed out that the method of block decomposition for functions was invented by Taibleson and Weiss [22] in the study of the convergence of Fourier series. Later on, many applications of the block decomposition to harmonic analysis were discovered (see [1], [12], [13], [14], [16], [18], [20] etc.). For further background and information about the theory of spaces generated by blocks and its applications to harmonic analysis, one can consult the book [15]. We remark that some ideas in the proof of our main results are taken from [9], [17] and our previous result [16], and our methods and techniques are more delicate and complex than those in [9], [17]. Our main results can be formulated as follows.

THEOREM 1. Suppose that $\Omega$ is homogeneous of degree zero on $\boldsymbol{R}^{n}$, and A has derivatives of order $m(|m| \geq 1)$ in $\operatorname{BMO}\left(\boldsymbol{R}^{n}\right)$. If $\Omega \in B_{q}^{0,1}\left(S^{n-1}\right)$ for some $q>1$, then for $1<p<\infty$, the following two facts are equivalent:

(i) If $P(x, y)$ is a non-degenerate polynomial having property $\mathcal{P}$, then

$$
\left\|T^{A} f\right\|_{p} \leq C(n, p, \operatorname{deg} P) \sum_{|\gamma|=m}\left\|D^{\gamma} A\right\|_{\mathrm{BMO}}\|f\|_{p} .
$$

(ii) The truncated operator

$$
S^{A} f(x)=\text { p.v. } \int_{|x-y|<1} \frac{\Omega(x-y)}{|x-y|^{n+m}} R_{m+1}(A ; x, y) f(y) d y
$$

satisfies

$$
\left\|S^{A} f\right\|_{p} \leq C(n, p) \sum_{|\gamma|=m}\left\|D^{\gamma} A\right\|_{\mathrm{BMO}}\|f\|_{p} .
$$

Here $\operatorname{deg} P$ denotes the total degree of $P(x, y)$.

For the general polynomial phase, we have the following result.

THEOREM 2. Under the same assumptions as in Theorem 1 about $A, p$ and $\Omega$, if the multilinear singular integral operator

$$
\bar{T}^{A} f(x)=\text { p.v. } \int_{\boldsymbol{R}^{n}} \frac{\Omega(x-y)}{|x-y|^{n+m}} R_{m+1}(A ; x, y) f(y) d y
$$

is bounded on $L^{p}\left(\boldsymbol{R}^{n}\right)$ with bound $C(n, p) \sum_{|\gamma|=m}\left\|D^{\gamma} A\right\|_{\mathrm{BMO}}$, then so is $T^{A}$ for any realvalued polynomial $P(x, y)$, with bound $C(n, p, \operatorname{deg} P) \sum_{|\gamma|=m}\left\|D^{\gamma} A\right\|_{\text {BMO. }}$

REMARK 1. If $\Omega \in B_{q}^{0,0}\left(S^{n-1}\right)$ for $q>1$, we need to impose some restrictions on the BMO functions. The corresponding results will be given in Section 5 .

This paper is organized as follows. In Section 2, we will give some preliminary lemmas. Next we will prove Theorem 2 in Section 3. The proof of Theorem 1 will be given in Section 4. Finally, we will give some further results without proofs in Section 5. Throughout this paper, we always use the letter $C$ to denote a positive constant that may vary at each occurrence but is independent of the essential variable.

The authors express their deep thanks to the referee for his many valuable comments including the simplified proof of (3.11). 


\section{Some Lemmas.}

LEMMA 1 (cf. [5]). Let $b(x)$ be a function on $\boldsymbol{R}^{n}$ with $m$-th order derivatives in $L^{t}\left(\boldsymbol{R}^{n}\right)$ for some $t, n<t \leq \infty$. Then

$$
\left|R_{m}(b ; x, y)\right| \leq C_{m, n}|x-y|^{m} \sum_{|\alpha|=m}\left(\frac{1}{\left|I_{x}^{y}\right|} \int_{I_{x}^{y}}\left|D^{\alpha} b(z)\right|^{t} d z\right)^{1 / t},
$$

where $I_{x}^{y}$ is the cube centered at $x$, with the sides parallel to the axes and with the diameter $5 \sqrt{n}|x-y|$.

Lemma 2. Let $\Omega$ be homogeneous of degree of zero on $\boldsymbol{R}^{n}$, A the same as that in Theorem 1 , and $1<p<\infty$. If $\Omega \in B_{q}^{0,0}\left(S^{n-1}\right)$ for some $q>1$, then the maximal operator

$$
M_{\Omega}^{A} f(x)=\sup _{r>0} r^{-(n+m)} \int_{|x-y|<r}\left|\Omega(x-y) R_{m+1}(A ; x, y) f(y)\right| d y
$$

is bounded on $L^{p}\left(\boldsymbol{R}^{n}\right)$ with bound $C \sum_{|\gamma|=m}\left\|D^{\gamma} A\right\|_{\mathrm{BMO}}$.

Proof. Obviously, it suffices to prove the lemma for $\tilde{M}_{\Omega}^{A}$, a variant of $M_{\Omega}^{A}$ defined by

$$
\tilde{M}_{\Omega}^{A} f(x):=\sup _{r>0} r^{-(n+m)} \int_{r / 2<|x-y|<r}\left|\Omega(x-y) R_{m+1}(A ; x, y) f(y)\right| d y .
$$

For fixed $x \in \boldsymbol{R}^{n}, r>0$, let $I(x, r)$ be the cube centered at $x$ and having side length $r$. Set

$$
\tilde{A}(y)=A(y)-\sum_{|\alpha|=m} \frac{1}{\alpha !} m_{I(x, r)}\left(D^{\alpha} A\right) y^{\alpha},
$$

where $m_{I(x, r)}\left(D^{\alpha} A\right)$ denotes the mean value of $D^{\alpha} A$ on $I(x, r)$. Note that for each fixed $\alpha$ with $|\alpha|=m$, we have $D^{\beta} y^{\alpha}=0$ if $|\beta| \geq m+1$. Thus

$$
R_{m+1}\left((\cdot)^{\alpha} ; x, y\right)=x^{\alpha}-\sum_{|\beta| \leq m} \frac{1}{\beta !} D^{\beta}\left(y^{\alpha}\right)(x-y)^{\beta}=0, \quad|\alpha|=m,
$$

which implies that

$R_{m+1}(\tilde{A} ; x, y)=R_{m+1}(A ; x, y)-\sum_{|\alpha|=m} \frac{1}{\alpha !} m_{I(x, r)}\left(D^{\alpha} A\right) R_{m+1}\left((\cdot)^{\alpha} ; x, y\right)=R_{m+1}(A ; x, y)$.

Since $\Omega \in B_{q}^{0,0}\left(S^{n-1}\right)$, we know by Definition 3 that $\Omega\left(x^{\prime}\right)=\sum_{s} C_{s} b_{s}\left(x^{\prime}\right)$, where each $b_{s}$ is a $q$-block, supported in $Q_{s}$ and

$$
\sum_{s}\left|C_{s}\right|\left(1+\log ^{+} \frac{1}{\left|Q_{s}\right|}\right)<\infty .
$$

Thus

$$
\begin{aligned}
\tilde{M}_{\Omega}^{A} f(x) & \leq \sum_{s}\left|C_{s}\right| \sup _{r>0} r^{-n-m} \int_{r / 2<|x-y|<r}\left|b_{s}(x-y) R_{m+1}(A ; x, y) f(y)\right| d y \\
& :=\sum_{s}\left|C_{S}\right| \tilde{M}_{b_{s}}^{A} f(x) .
\end{aligned}
$$


Now, we estimate $\tilde{M}_{b_{s}}^{A}$. For any $1<\lambda \leq q$, let $\lambda^{\prime}=\lambda /(\lambda-1)$. Applying Hölder's inequality, we have

$$
\begin{aligned}
\tilde{M}_{b_{s}}^{A} f(x) \leq & \sup _{r>0}\left(r^{-n} \int_{|x-y|<r}\left|b_{s}(x-y)\right|^{\lambda}|f(y)| d y\right)^{1 / \lambda} \\
& \times \sup _{r>0}\left(r^{-n-m \lambda^{\prime}} \int_{r / 2<|x-y|<r}\left|R_{m+1}(\tilde{A} ; x, y)\right|^{\lambda^{\prime}}|f(y)| d y\right)^{1 / \lambda^{\prime}} \\
:= & I(f)(x)^{1 / \lambda} I I(f)(x)^{1 / \lambda^{\prime}} .
\end{aligned}
$$

Observe that $I(f)(x)=M_{\left|b_{s}\right|}(f)(x)$. By the method of rotation of Calderón and Zygmund [2], we obtain

$$
\|I(f)\|_{p} \leq C\left\|b_{s}\right\|_{L^{\lambda}\left(S^{n-1}\right)}^{\lambda}\|f\|_{p}, \quad 1<p<\infty .
$$

On the other hand, by Lemma 1 and the same argument as that in the proof of Lemma 2 in [4], we can deduce that for $1<t<p$,

$$
\|I I(f)\|_{p} \leq C_{1} C_{2}^{\lambda^{\prime}} \Gamma\left(\lambda^{\prime} t^{\prime}+1\right)^{1 / t^{\prime}} \sum_{|\alpha|=m}\left\|D^{\alpha} A\right\|_{\mathrm{BMO}}^{\lambda^{\prime}}\|f\|_{p}, \quad 1<p<\infty,
$$

where $\Gamma(u)=\int_{0}^{\infty} e^{-v} v^{u-1} d v, C_{1}$ and $C_{2}$ are constants depending only on $n$. Here we use the result (see [11]) that if $a \in \mathrm{BMO}\left(\boldsymbol{R}^{n}\right)$, then for any cube $Q$ there exist constants $C_{1}$ and $C_{2}$ depending only on $n$ such that

$$
\frac{1}{|Q|} \int_{Q}\left|a(x)-a_{Q}\right|^{\lambda} d x \leq C_{1} C_{2}^{\lambda} \Gamma(\lambda+1)\|a\|_{\mathrm{BMO}\left(\boldsymbol{R}^{n}\right)}^{\lambda} .
$$

Combining the estimates for the terms I and II, we obtain by another application of Hölder's inequality that

$$
\left\|\tilde{M}_{b_{s}}^{A}(f)\right\|_{p} \leq C \lambda^{\prime}\left\|b_{s}\right\|_{L^{\lambda}\left(S^{n-1}\right)} \sum_{|\gamma|=m}\left\|D^{\gamma} A\right\|_{\mathrm{BMO}}\|f\|_{p}, \quad 1<p<\infty,
$$

where $C$ is independent of $\lambda$.

Recalling that for each $b_{s}, \operatorname{supp}\left(b_{s}\right) \subset Q_{s}$ and $\left\|b_{s}\right\|_{L^{q}\left(S^{n-1}\right)} \leq\left|Q_{s}\right|^{1 / q-1}$, if $\left|Q_{s}\right| \geq$ $e^{q /(1-q)}$, we take $\lambda=q$ and obtain

$$
\begin{aligned}
\left\|\tilde{M}_{b_{s}}^{A} f\right\|_{p} & \leq C q^{\prime}\left\|b_{s}\right\|_{L^{q}\left(S^{n-1}\right)} \sum_{|\gamma|=m}\left\|D^{\gamma} A\right\|_{\mathrm{BMO}}\|f\|_{p} \\
& \leq C\left|Q_{s}\right|^{1 / q-1} \sum_{|\gamma|=m}\left\|D^{\gamma} A\right\|_{\mathrm{BMO}}\|f\|_{p} \\
& \leq C \sum_{|\gamma|=m}\left\|D^{\gamma} A\right\|_{\mathrm{BMO}}\|f\|_{p}
\end{aligned}
$$


if $\left|Q_{s}\right|<e^{q / 1-q}$, we take $\lambda=\log \left|Q_{s}\right| /\left(1+\log \left|Q_{s}\right|\right)$, then $1<\lambda<q, \lambda^{\prime}=\log \left(1 /\left|Q_{s}\right|\right)$ and

$$
\begin{aligned}
\left\|\tilde{M}_{b_{s}}^{A} f\right\|_{p} & \leq C \lambda^{\prime}\left\|b_{s}\right\|_{L^{\lambda}\left(S^{n-1}\right)} \sum_{|\gamma|=m}\left\|D^{\gamma} A\right\|_{\mathrm{BMO}}\|f\|_{p} \\
& \leq C \log \frac{1}{\left|Q_{s}\right|}\left\|b_{s}\right\|_{L^{q}\left(S^{n-1}\right)}\left|Q_{s}\right|^{1 / \lambda-1 / q} \sum_{|\gamma|=m}\left\|D^{\gamma} A\right\|_{\mathrm{BMO}}\|f\|_{p} \\
& \leq C \log \frac{1}{\left|Q_{s}\right|}\left|Q_{s}\right|^{1 / \lambda-1} \sum_{|\gamma|=m}\left\|D^{\gamma} A\right\|_{\mathrm{BMO}}\|f\|_{p} \\
& =C \log \frac{1}{\left|Q_{s}\right|}\left|Q_{s}\right|^{1 / \log \left|Q_{s}\right|} \sum_{|\gamma|=m}\left\|D^{\gamma} A\right\|_{\mathrm{BMO}}\|f\|_{p} \\
& \leq C \log \frac{1}{\left|Q_{s}\right|} \sum_{|\gamma|=m}\left\|D^{\gamma} A\right\|_{\mathrm{BMO}}\|f\|_{p} .
\end{aligned}
$$

Consequently, for each $b_{s}$, we get that

$$
\left\|\tilde{M}_{b_{s}}^{A} f\right\|_{p} \leq C\left(1+\log \frac{1}{\left|Q_{s}\right|}\right) \sum_{|\gamma|=m}\left\|D^{\gamma} A\right\|_{\mathrm{BMO}}\|f\|_{p} .
$$

By the last inequality and (2.1), we obtain

$$
\begin{aligned}
\left\|\tilde{M}_{\Omega}^{A} f\right\|_{p} & \leq C \sum_{s}\left|C_{s}\right|\left(1+\log ^{+} \frac{1}{\left|Q_{s}\right|}\right) \sum_{|\gamma|=m}\left\|D^{\gamma} A\right\|_{\mathrm{BMO}}\|f\|_{p} \\
& \leq C \sum_{|\gamma|=m}\left\|D^{\gamma} A\right\|_{\mathrm{BMO}}\|f\|_{p},
\end{aligned}
$$

which proves Lemma 2.

LEMMA 3. Suppose that $K(x, y)$ is a distribution which agree with a function away from the diagonal $\{x=y\}$ satisfying

$$
|K(x, y)| \leq \frac{|\Omega(x-y)|}{|x-y|^{n+m}}\left|R_{m+1}(A ; x, y)\right|,
$$

with $\Omega, R_{m+1}(A ; x, y)$ as in Lemma 2. If the operator

$$
T f(x)=\text { p.v. } \int_{\boldsymbol{R}^{n}} K(x, y) f(y) d y
$$

is bounded on $L^{p}\left(\boldsymbol{R}^{n}\right)$, then the truncated operator

$$
T_{0} f(x)=\text { p.v. } \int_{|x-y|<\varepsilon} K(x, y) f(y) d y
$$

is also bounded on $L^{p}\left(\boldsymbol{R}^{n}\right)$ with bound $C\left(\|T\|+\sum_{|\gamma|=m}\left\|D^{\gamma} A\right\|_{\mathrm{BMO}}\right)$, where $C$ is independent of $\varepsilon$ and $T$.

This lemma can be proved by Lemma 2 and the same argument as that used in [9, p. 54]. We omit the details for brevity. 
3. Proof of Theorem 2. We shall carry out the argument by a double induction on the degrees in $x$ and $y$ of the polynomial. By the $L^{p}\left(\boldsymbol{R}^{n}\right)$ boundedness of $\bar{T}^{A}$, it is obvious that Theorem 2 holds if the polynomial is trivial. Let $k$ and $l$ be two positive integers and let the polynomial have degrees $k$ in $x$ and $l$ in $y$. We assume that Theorem 2 is known for all polynomials which are sums of monomials of degree less than or equal to $k-1$ in $x$ times monomials of any degree in $y$, together with monomials which are of degree $k$ in $x$ times monomials which are of degree less than or equal to $l-1$ in $y$.

Now, we proceed to the proof of the inductive step. Write

$$
P(x, y)=\sum_{|\alpha|=k,|\beta|=l} a_{\alpha \beta} x^{\alpha} y^{\beta}+R_{0}(x, y),
$$

where $R_{0}(x, y)$ satisfies the above inductive assumption. By dilation-invariance, we may assume that $\sum_{|\alpha|=k,|\beta|=l}\left|a_{\alpha \beta}\right|=1$. Decompose $T^{A}$ as

$$
\begin{aligned}
T^{A} f(x)= & \int_{|x-y|<1} e^{i P(x, y)} \frac{\Omega(x-y)}{|x-y|^{n+m}} R_{m+1}(A ; x, y) f(y) d y \\
& \quad+\int_{|x-y| \geq 1} e^{i P(x, y)} \frac{\Omega(x-y)}{|x-y|^{n+m}} R_{m+1}(A ; x, y) f(y) d y \\
:= & T_{0}^{A} f(x)+T_{\infty}^{A} f(x) .
\end{aligned}
$$

For $T_{0}^{A}$, by Lemma 2, Lemma 3 and the same argument as that used in [9, p. 55-56], we have

$$
\left\|T_{0}^{A} f\right\|_{p} \leq C \sum_{|\gamma|=m}\left\|D^{\gamma} A\right\|_{\mathrm{BMO}}\|f\|_{p} .
$$

Now, we consider the operator $T_{\infty}^{A}$. By Definition 2, we have

$$
\begin{aligned}
T_{\infty}^{A} f(x) & =\sum_{j=0}^{\infty} \sum_{s} C_{s} \int_{2^{j} \leq|x-y|<2^{j+1}} e^{i P(x, y)} \frac{b_{s}(x-y)}{|x-y|^{n+m}} R_{m+1}(A ; x, y) f(y) d y \\
& :=\sum_{s} C_{s} \sum_{j=0}^{\infty} T_{j, s}^{A} f(x) .
\end{aligned}
$$

Thus for all $1<p<\infty$,

$$
\left\|T_{\infty}^{A} f\right\|_{p} \leq \sum_{s}\left|C_{s}\right| \sum_{j=0}^{\infty}\left\|T_{j, s}^{A} f\right\|_{p}
$$

We shall show that

$$
\sum_{j=0}^{\infty}\left\|T_{j, s}^{A} f\right\|_{p} \leq C\left\{1+\left(\log ^{+} \frac{1}{\left|Q_{s}\right|}\right)^{2}\right\} \sum_{|\gamma|=m}\left\|D^{\gamma} A\right\|_{\text {BMO }}\|f\|_{p} .
$$


By (2.2), we have for $1<\lambda \leq q$,

$$
\left\|T_{j, s}^{A} f\right\|_{p} \leq C \lambda^{\prime}\left\|b_{s}\right\|_{L^{\lambda}\left(S^{n-1}\right)} \sum_{|\gamma|=m}\left\|D^{\gamma} A\right\|_{\mathrm{BMO}}\|f\|_{p} .
$$

To prove (3.3), it suffices to prove that, for each $b_{s}$ and any $1<\lambda \leq q$,

$$
\left\|T_{j, s}^{A} f\right\|_{2} \leq C B^{\delta} 2^{-j \theta \delta} \lambda^{\prime}\left\|b_{s}\right\|_{L^{\lambda}\left(S^{n-1}\right)} \sum_{|\gamma|=m}\left\|D^{\gamma} A\right\|_{\mathrm{BMO}}\|f\|_{2}
$$

holds uniformly in $\delta \in(0,1]$ such that $\delta<\min \left(k / 2 l, k /(k+l) \lambda^{\prime}\right)$. Here $0<\theta<1$ and $C$, $B$ depend only on $n$ and, in the latter, $\operatorname{deg} P$.

If we can do this, then interpolation between (3.4) and (3.5) shows that for $1<p<\infty$

$$
\left\|T_{j, s}^{A} f\right\|_{p} \leq C B^{\theta_{1} \delta} 2^{-j \theta_{1} \theta \delta} \lambda^{\prime}\left\|b_{s}\right\|_{L^{\lambda}\left(S^{n-1}\right)} \sum_{|\gamma|=m}\left\|D^{\gamma} A\right\|_{\mathrm{BMO}}\|f\|_{p},
$$

where $0<\theta_{1} \leq 1$. Therefore, for each $b_{s}$, we consider the following two cases, respectively:

Case 1. When $\left|Q_{s}\right| \geq e^{q /(1-q)}$, we take $\lambda=q$. Then

$$
\begin{aligned}
\left\|T_{j, s}^{A} f\right\|_{p} & \leq C 2^{-j \theta_{1} \theta \delta}\left\|b_{s}\right\|_{L^{q}\left(S^{n-1}\right)} \sum_{|\gamma|=m}\left\|D^{\gamma} A\right\|_{\mathrm{BMO}}\|f\|_{p} \\
& \leq C 2^{-j \theta_{1} \theta \delta}\left|Q_{s}\right|^{1 / q-1} \sum_{|\gamma|=m}\left\|D^{\gamma} A\right\|_{\mathrm{BMO}}\|f\|_{p} \\
& \leq C 2^{-j \theta_{1} \theta \delta} \sum_{|\gamma|=m}\left\|D^{\gamma} A\right\|_{\mathrm{BMO}}\|f\|_{p} .
\end{aligned}
$$

So

$$
\sum_{j=0}^{\infty}\left\|T_{j, s}^{A} f\right\|_{p} \leq C \sum_{j=0}^{\infty} 2^{-j \theta_{1} \theta \delta} \sum_{|\gamma|=m}\left\|D^{\gamma} A\right\|_{\mathrm{BMO}}\|f\|_{p} \leq C \sum_{|\gamma|=m}\left\|D^{\gamma} A\right\|_{\mathrm{BMO}}\|f\|_{p} .
$$

Case 2. When $\left|Q_{s}\right|<e^{q /(1-q)}$, we take $\lambda=\log \left|Q_{s}\right| /\left(1+\log \left|Q_{s}\right|\right)$ and choose $\delta=$ $\sigma / \lambda^{\prime}<\min \left(k / 2 l, k /(k+l) \lambda^{\prime}\right)$, where $\sigma$ is a positive constant depending only on $k$ and $l$. Then

$$
\begin{aligned}
\left\|T_{j, s}^{A} f\right\|_{p} & \leq C B^{\theta_{1} \sigma / \lambda^{\prime}} 2^{-\theta_{1} \theta \sigma j / \lambda^{\prime}} \lambda^{\prime}\left\|b_{s}\right\|_{L^{\lambda}\left(S^{n-1}\right)} \sum_{|\gamma|=m}\left\|D^{\gamma} A\right\|_{\mathrm{BMO}}\|f\|_{p} \\
& \leq C B^{-\theta_{1} \sigma / \log \left|Q_{s}\right|} 2^{\theta_{1} \theta \sigma j / \log \left|Q_{s}\right|}\left|Q_{s}\right|^{1 / \log \left|Q_{s}\right|}\left(\log \frac{1}{\left|Q_{s}\right|}\right) \sum_{|\gamma|=m}\left\|D^{\gamma} A\right\|_{\mathrm{BMO}}\|f\|_{p} \\
& \leq C B^{-\theta_{1} \sigma / \log \left|Q_{s}\right|} 2^{\theta_{1} \theta \sigma j / \log \left|Q_{s}\right|}\left(\log \frac{1}{\left|Q_{s}\right|}\right) \sum_{|\gamma|=m}\left\|D^{\gamma} A\right\|_{\mathrm{BMO}}\|f\|_{p} .
\end{aligned}
$$

Since $\left|Q_{S}\right|<e^{q /(1-q)}$, we have $\log \left|Q_{s}\right|<q /(1-q)$, i.e., $-\log \left|Q_{s}\right|>q /(q-1)>1$. Consequently, if $B \leq 1$, then $B^{-\theta_{1} \sigma / \log \left|Q_{s}\right|} \leq 1$; if $B>1$, then $B^{-\theta_{1} \sigma / \log \left|Q_{s}\right|}<B^{\theta_{1} \sigma(q-1) / q}=C$. 
Hence

$$
\left\|T_{j, s}^{A} f\right\|_{p} \leq C 2^{\theta_{1} \theta \sigma j / \log \left|Q_{s}\right|} \log \frac{1}{\left|Q_{s}\right|} \sum_{|\gamma|=m}\left\|D^{\gamma} A\right\|_{\mathrm{BMO}}\|f\|_{p} .
$$

So

$$
\begin{aligned}
\sum_{j=0}^{\infty}\left\|T_{j, s}^{A} f\right\|_{p} & \leq C \sum_{j=0}^{\infty} 2^{\theta_{1} \theta \sigma j / \log \left|Q_{s}\right|} \log \frac{1}{\left|Q_{s}\right|} \sum_{|\gamma|=m}\left\|D^{\gamma} A\right\|_{\mathrm{BMO}}\|f\|_{p} \\
& \leq C\left(\log \frac{1}{\left|Q_{s}\right|}\right)^{2} \sum_{|\gamma|=m}\left\|D^{\gamma} A\right\|_{\mathrm{BMO}}\|f\|_{p} .
\end{aligned}
$$

This shows that (3.3) hold. Consequently, by (3.2), we obtain

$$
\begin{aligned}
\left\|T_{\infty}^{A} f\right\|_{p} & \leq C \sum_{s}\left|C_{s}\right|\left\{1+\left(\log ^{+} \frac{1}{\left|Q_{s}\right|}\right)^{2}\right\} \sum_{|\gamma|=m}\left\|D^{\gamma} A\right\|_{\mathrm{BMO}}\|f\|_{p} \\
& \leq C \sum_{|\gamma|=m}\left\|D^{\gamma} A\right\|_{\mathrm{BMO}}\|f\|_{p} .
\end{aligned}
$$

Summarizing (3.1) and (3.7), we conclude the proof of Theorem 2.

Now we return to the proof of (3.5). To do this, we turn our attention to the operator

$$
\tilde{T}_{j, s}^{A} f(x)=\int_{1<|x-y| \leq 2} e^{i P\left(2^{j} x, 2^{j} y\right)} \frac{b_{s}(x-y)}{|x-y|^{n+m}} R_{m+1}(A ; x, y) f(y) d y .
$$

By dilation-invariance, it is easy to see that the proof of (3.5) can be reduced to showing that

$$
\left\|\tilde{T}_{j, s}^{A} f\right\|_{2} \leq C B^{\delta} 2^{-j \theta \delta} \lambda^{\prime}\left\|b_{s}\right\|_{L^{\lambda}\left(S^{n-1}\right)} \sum_{|\gamma|=m}\left\|D^{\gamma} A\right\|_{\mathrm{BMO}}\|f\|_{2},
$$

where $C$ and $B$ are as those in (3.5).

Decompose $\boldsymbol{R}^{n}$ into $\boldsymbol{R}^{n}=\bigcup_{d} I_{d}$, where $I_{d}$ is a cube with side length 1 and the cubes have disjoint interiors. Set $f_{d}=f \chi_{I_{d}}$. Since the support of $\tilde{T}_{j, s}^{A} f_{d}$ is contained in a fixed multiple of $I_{d}$, the supports of the various terms $\tilde{T}_{j, s}^{A} f_{d}$ have bounded overlaps. Thus

$$
\left\|\tilde{T}_{j, s}^{A} f\right\|_{2}^{2} \leq C \sum_{d}\left\|\tilde{T}_{j, s}^{A} f_{d}\right\|_{2}^{2} .
$$

For each fixed $d$, denote $\tilde{I}_{d}=10 n I_{d}$. Let $\Psi_{d}(x) \in C_{0}^{\infty}\left(\boldsymbol{R}^{n}\right)$ such that $0 \leq \Psi_{d} \leq 1, \Psi_{d}$ is identically one on $4 \sqrt{n} I_{d}$ and vanishes outsides of $6 \sqrt{n} I_{d},\left\|D^{\alpha} \Psi_{d}\right\|_{\infty} \leq C_{\alpha}$ for all multiindex $\alpha$. Let $x_{0}$ be a point on the boundary of $8 \sqrt{n} I_{d}$. Denote

$$
A^{\Psi_{d}}(y)=R_{m}\left(A(\cdot)-\sum_{|\beta|=m} \frac{1}{\beta !} m_{\tilde{I}_{d}}\left(D^{\beta} A\right)(\cdot)^{\beta} ; y, x_{0}\right) \Psi_{d}(y)
$$

and for multi-index $\alpha$, define

$$
\tilde{T}_{j, s}^{\alpha} h(x)=\int_{1<|x-y| \leq 2} e^{i P\left(2^{j} x, 2^{j} y\right)} \frac{b_{s}(x-y)}{|x-y|^{n+m}}(x-y)^{\alpha} h(y) d y .
$$


It is easy to see that

$$
\begin{aligned}
\tilde{T}_{j, s}^{A} f_{d}(x)= & \tilde{T}_{j, s}^{A^{\Psi_{d}}} f_{d}(x)=A^{\Psi_{d}}(x) \tilde{T}_{j, s}^{0} f_{d}(x) \\
& -\sum_{|\alpha|<m} \frac{1}{\alpha !} \tilde{T}_{j, s}^{\alpha}\left(D^{\alpha} A^{\Psi_{d}} f_{d}\right)(x)-\sum_{|\alpha|=m} \frac{1}{\alpha !} \tilde{T}_{j, s}^{\alpha}\left(D^{\alpha} A^{\Psi_{d}} f_{d}\right)(x) \\
:= & G_{1}+G_{2}+G_{3} .
\end{aligned}
$$

To estimate these terms, we shall use the following lemma.

LEMMA 4. For any multi-index $\alpha$ and $\lambda>1$,

$$
\left\|\tilde{T}_{j, s}^{\alpha} h\right\|_{p} \leq C B^{\delta} 2^{-\theta \delta j}\left\|b_{s}\right\|_{L^{\lambda}\left(S^{n-1}\right)}\|h\|_{p}, \quad 1<p<\infty
$$

holds uniformly for $\delta \in(0,1]$ such that $\delta<\min \left(k / 2 l, k /(k+l) \lambda^{\prime}\right)$. Here, $C, B$ and $\theta$ are as those in (3.5).

Proof. Let $b(r)=r^{|\alpha|-m}$ and $\tilde{b}_{s}(x)=b_{s}(x)(x /|x|)^{\alpha}$. It is obvious that $\tilde{b}_{s}(x)$ is homogeneous of degree zero and $\left|\tilde{b}_{s}(x)\right|=\left|b_{s}(x)\right|$. Note that

$$
\begin{aligned}
& \int_{2^{j}<|x-y| \leq 2^{j+1}} e^{i P(x, y)} \frac{b_{s}(x-y)}{|x-y|^{n+m}}(x-y)^{\alpha} h(y) d y \\
& \quad=\int_{2^{j}<|x-y| \leq 2^{j+1}} e^{i P(x, y)} \frac{\tilde{b}_{s}(x-y)}{|x-y|^{n}} b(|x-y|) h(y) d y .
\end{aligned}
$$

Using Proposition 2 and Proposition 3 in [16], by the same argument as that used in [17, p. 209-213], we can find that, for any $1<\lambda \leq \infty$,

$$
\begin{gathered}
\left\|\int_{2^{j}<|\cdot-y| \leq 2^{j+1}} e^{i P(\cdot, y)} \frac{\tilde{b}_{s}(\cdot-y)}{|\cdot-y|^{n}} b(|\cdot-y|) h(y) d y\right\|_{p} \\
\leq C\|b\|_{\infty,\left[2^{j}, 2^{j+1}\right]} B^{\delta} 2^{-\theta \delta j}\left\|\tilde{b}_{s}\right\|_{L^{\lambda}\left(S^{n-1}\right)}\|h\|_{p} \\
\leq C B^{\delta} 2^{-(\theta \delta+m-|\alpha|) j}\left\|b_{S}\right\|_{L^{\lambda}\left(S^{n-1}\right)}\|h\|_{p} .
\end{gathered}
$$

This leads to the conclusion of Lemma 4.

We now return to the estimates of $G_{1}, G_{2}, G_{3}$. Noticing that for multi-index $\alpha$ and $|\alpha| \leq m$, we have (see [5, p. 452])

$$
D^{\alpha} A^{\Psi_{d}}(y)=\sum_{\alpha=u+v} C_{u, v} R_{m-|u|}\left(D^{u}\left(A(\cdot)-\sum_{|\beta|=m} \frac{1}{\beta !} m_{\tilde{I}_{d}}\left(D^{\beta} A\right)(\cdot)^{\beta}\right) ; y, x_{0}\right) D^{v} \Psi_{d}(y) .
$$

Since $\operatorname{supp} \Psi_{d} \subseteq 6 \sqrt{n} I_{d}$, by Lemma 1 , we have

$$
\left|D^{\alpha} A^{\Psi_{d}}(y)\right| \leq C \sum_{|\beta|=m}\left(\frac{1}{\left|\tilde{I}_{x_{0}}^{y}\right|} \int_{\tilde{I}_{x_{0}}^{y}}\left|D^{\beta} A(z)-m_{\tilde{I}_{d}}\left(D^{\beta} A\right)\right|^{t} d_{z}\right)^{1 / t} \leq C \sum_{|\gamma|=m}\left\|D^{\gamma} A\right\|_{\mathrm{BMO}},
$$

where $n<t<\infty$. So by Lemma 4 ,

$$
\left\|G_{1}\right\|_{2} \leq\left\|A^{\Psi_{d}}\right\|_{\infty}\left\|\tilde{T}_{j, s}^{0} f_{d}\right\|_{2} \leq C \sum_{|\gamma|=m}\left\|D^{\gamma} A\right\|_{\mathrm{BMO}} B^{\delta} 2^{-\theta \delta j}\left\|b_{s}\right\|_{L^{\lambda}\left(S^{n-1}\right)}\left\|f_{d}\right\|_{2} .
$$


Similarly,

$$
\begin{aligned}
\left\|G_{2}\right\|_{2} & \leq C \sum_{|\alpha|<m}\left\|\tilde{T}_{j, s}^{\alpha}\left(D^{\alpha} A^{\Psi_{d}} f_{d}\right)\right\|_{2} \\
& \leq C B^{\delta} 2^{-\theta \delta j}\left\|b_{s}\right\|_{L^{\lambda}\left(S^{n-1}\right)} \sum_{|\alpha|<m}\left\|D^{\alpha} A^{\Psi_{d}}\right\|_{\infty}\left\|f_{d}\right\|_{2} \\
& \leq C B^{\delta} 2^{-\theta \delta j}\left\|b_{s}\right\|_{L^{\lambda}\left(S^{n-1}\right)} \sum_{|\gamma|=m}\left\|D^{\gamma} A\right\|_{\mathrm{BMO}}\left\|f_{d}\right\|_{2} .
\end{aligned}
$$

It remains to deal with the term $G_{3}$. Let $\lambda^{\prime}<p<\infty, 0<\rho<\infty$ and $\chi(x, y)$ be the characteristic function of the set $\left\{(x, y) \in \boldsymbol{R}^{n} \times \boldsymbol{R}^{n}: 1<|x-y| \leq 2\right\}$. Then

$$
\begin{aligned}
\left|\tilde{T}_{j, s}^{\alpha} h(x)\right| & \leq C \int_{1<|x-y| \leq 2}\left|b_{s}(x-y) h(y)\right| d y \\
& \leq C\left\|b_{s}\right\|_{L^{\lambda}\left(S^{n-1}\right)}\left(\int_{1<|x-y| \leq 2}|h(y)|^{\lambda^{\prime}} d y\right)^{1 / \lambda^{\prime}} \\
& \leq C\left\|b_{s}\right\|_{L^{\lambda}\left(S^{n-1}\right)}\left(\int_{\boldsymbol{R}^{n}}|\chi(x, y) h(y)|^{p} d y\right)^{1 / p} .
\end{aligned}
$$

Hence

$$
\begin{aligned}
\left\|\tilde{T}_{j, s}^{\alpha} h\right\|_{p+\rho} & \leq C\left\|b_{S}\right\|_{L^{\lambda}\left(S^{n-1}\right)}\left(\int_{\boldsymbol{R}^{n}}\|\chi(\cdot, y) h(y)\|_{p+\rho}^{p} d y\right)^{1 / p} \\
& \leq C\left\|b_{S}\right\|_{L^{\lambda}\left(S^{n-1}\right)}\|h\|_{p} .
\end{aligned}
$$

Consequently,

$$
\left\|\tilde{T}_{j, s}^{\alpha} h\right\|_{2 \lambda^{\prime}+2} \leq C\left\|b_{s}\right\|_{L^{\lambda}\left(S^{n-1}\right)}\|h\|_{\lambda^{\prime}+2} .
$$

Invoking Lemma 4, we have

$$
\left\|\tilde{T}_{j, s}^{\alpha} h\right\|_{3 / 2} \leq C B^{\delta} 2^{-\theta \delta j}\left\|b_{s}\right\|_{L^{\lambda}\left(S^{n-1}\right)}\|h\|_{3 / 2} .
$$

Interpolating between (3.12) and (3.13) gives

$$
\left\|\tilde{T}_{j, s}^{\alpha} h\right\|_{2} \leq C B^{\theta_{2} \delta} 2^{-\theta_{2} \theta \delta j}\left\|b_{s}\right\|_{L^{\lambda}\left(S^{n-1}\right)}\|h\|_{2-\rho},
$$

where $\theta_{2}=3 \lambda^{\prime} /\left(4 \lambda^{\prime}+1\right), \rho=\lambda^{\prime} /\left(2 \lambda^{\prime 2}+5 \lambda^{\prime}+1\right)$.

On the other hand, (3.10) and Lemma 1 show that if $|\alpha|=m$, then

$$
\begin{aligned}
\left|D^{\alpha} A^{\Psi_{d}}(y)\right| & \leq \sum_{\alpha=u+v,|u|<m} C_{u, v}\left|R_{m-|u|}\left(D^{u} A(\cdot)-\sum_{|\beta|=m} \frac{1}{\beta !} m_{\tilde{I}_{d}}\left(D^{\beta} A\right)(\cdot)^{\beta} ; y, x_{0}\right) D^{v} \Psi_{d}(y)\right| \\
& +\sum_{|u|=m}\left|\left(D^{u} A(y)-m_{\tilde{I}_{d}}\left(D^{u} A\right)\right) \Psi_{d}(y)\right| \\
\leq & C\left(\sum_{|\gamma|=m}\left\|D^{\gamma} A\right\|_{\mathrm{BMO}}+\sum_{|\gamma|=m}\left|D^{\gamma} A(y)-m_{\tilde{I}_{d}}\left(D^{\gamma} A\right)\right|\right) .
\end{aligned}
$$


Thus, for any $1<t<\infty$,

$$
\left\|D^{\alpha} A^{\Psi_{d}}\right\|_{t} \leq C t \sum_{|\gamma|=m}\left\|D^{\gamma} A\right\|_{\mathrm{BMO}} .
$$

For $\rho$ in (3.14), choose $t=8 \lambda^{\prime}+18+4 / \lambda^{\prime} \leq 30 \lambda^{\prime}$ such that $1 / 2+1 / t=1 /(2-\rho)$, it follows from (3.14) that

$$
\begin{aligned}
\left\|G_{3}\right\|_{2} & \leq C \sum_{|\alpha|=m}\left\|\tilde{T}_{j, s}^{\alpha}\left(D^{\alpha} A^{\Psi_{d}} f_{d}\right)\right\|_{2} \leq C B^{\theta_{2} \delta} 2^{-\theta_{2} \theta \delta j}\left\|b_{s}\right\|_{L^{\lambda}\left(S^{n-1}\right)} \sum_{|\alpha|=m}\left\|D^{\alpha} A^{\Psi_{d}} f_{d}\right\|_{2-\rho} \\
& \leq C B^{\theta_{2} \delta} 2^{-\theta_{2} \theta \delta j}\left\|b_{s}\right\|_{L^{\lambda}\left(S^{n-1}\right)} \sum_{|\alpha|=m}\left\|D^{\alpha} A^{\Psi_{d}}\right\|_{t}\left\|f_{d}\right\|_{2} \\
& \leq C B^{\theta_{2} \delta} 2^{-\theta_{2} \theta \delta j}\left\|b_{s}\right\|_{L^{\lambda}\left(S^{n-1}\right)} t \sum_{|\alpha|=m}\left\|D^{\alpha} A\right\|_{\mathrm{BMO}}\left\|f_{d}\right\|_{2} \\
& \leq C B^{\delta} 2^{-\theta \delta j} \lambda^{\prime}\left\|b_{S}\right\|_{L^{\lambda}\left(S^{n-1}\right)} \sum_{|\gamma|=m}\left\|D^{\gamma} A\right\|_{\mathrm{BMO}}\left\|f_{d}\right\|_{2} .
\end{aligned}
$$

This finishes the proof of Theorem 2 .

4. Proof of Theorem 1. (i) implies (ii): Suppose that $P(x, y)$ has the property $\mathcal{P}$. Decompose

$$
\begin{aligned}
T^{A} f(x)= & \int_{|x-y|<1} e^{i P(x, y)} \frac{\Omega(x-y)}{|x-y|^{n+m}} R_{m+1}(A ; x, y) f(y) d y \\
& +\int_{|x-y| \geq 1} e^{i P(x, y)} \frac{\Omega(x-y)}{|x-y|^{n+m}} R_{m+1}(A ; x, y) f(y) d y \\
:= & T_{0}^{A} f(x)+T_{\infty}^{A} f(x) .
\end{aligned}
$$

By Lemma 3, we know that $T_{0}^{A}$ is bounded on $L^{p}\left(\boldsymbol{R}^{n}\right)$. Taking an $h \in \boldsymbol{R}^{n}$, for $|x-h|<1$, we have

$$
T_{0}^{A} f(x)=T_{0}^{A}\left[f(\cdot) \chi_{B(h, 2)}(\cdot)\right](x),
$$

where $B(h, 2)=\{y:|y-h|<2\}$. Thus

(4.1) $\quad\left(\int_{|x-h|<1}\left|T_{0}^{A} f(x)\right|^{p} d x\right)^{1 / p} \leq C \sum_{|\gamma|=m}\left\|D^{\gamma} A\right\|_{\mathrm{BMO}}\left(\int_{|y-h|<2}|f(y)|^{p} d y\right)^{1 / p}$.

Since $P(x, y)$ has the property $\mathcal{P}$, we write

$$
\begin{aligned}
S^{A} f(x) & =\int_{|x-y|<1} \frac{\Omega(x-y)}{|x-y|^{n+m}} R_{m+1}(A ; x, y) f(y) d y \\
& =e^{i R_{0}(x, h)} \int_{|x-y|<1} e^{i P(x, y)} \frac{\Omega(x-y)}{|x-y|^{n+m}} R_{m+1}(A ; x, y) e^{-i P(x-h, y-h)} e^{-i R_{1}(y, h)} f(y) d y .
\end{aligned}
$$


Observe that Taylor's expression of $e^{-i P(x-h, y-h)}$ is

$$
\begin{aligned}
e^{-i P(x-h, y-h)} & =\sum_{k=0}^{\infty} \frac{(-i)^{k}}{k !}\left[\sum_{\alpha, \beta} a_{\alpha \beta}(x-h)^{\alpha}(y-h)^{\beta}\right]^{k} \\
& =\sum_{k=0}^{\infty} \frac{(-i)^{k}}{k !} \sum_{u, v} a_{u v}^{\prime}(x-h)^{u}(y-h)^{v}
\end{aligned}
$$

Hence

$$
\begin{aligned}
\left(\int_{|x-h|<1}\left|S^{A} f(x)\right|^{p} d x\right)^{1 / p} & \\
\leq & \sum_{k=0}^{\infty} \frac{1}{k !} \sum_{u, v}\left|a_{u v}^{\prime}\right|\left(\int_{|x-h|<1}\left|(x-h)^{u}\right|\right. \\
& \left.\times\left|\int_{|x-y|<1} e^{i P(x, y)} \frac{\Omega(x-y)}{|x-y|^{n+m}} e^{-i R_{1}(y, h)}(y-h)^{v} f(y) d y\right|^{p} d x\right)^{1 / p} \\
\leq & \sum_{k=0}^{\infty} \frac{1}{k !} \sum_{u, v}\left|a_{u v}^{\prime}\right|\left|\xi^{u}\right|\left(\int_{|x-h|<1}\left|T_{0}^{A}\left[e^{-i R_{1}(\cdot, h)}(\cdot-h)^{v} f(\cdot)\right](x)\right|^{p} d x\right)^{1 / p}
\end{aligned}
$$

where $\xi=(1,1, \ldots, 1)$. By (4.1), we have

$$
\begin{aligned}
& \left(\int_{|x-h|<1}\left|S^{A} f(x)\right|^{p} d x\right)^{1 / p} \\
& \quad \leq C \sum_{k=0}^{\infty} \frac{1}{k !} \sum_{u, v}\left|a_{u v}^{\prime}\right|\left|\xi^{u}\right| \sum_{|\gamma|=m}\left\|D^{\gamma} A\right\|_{\mathrm{BMO}}\left(\int_{|y-h|<2}\left|f(y)(y-h)^{v}\right|^{p} d x\right)^{1 / p} \\
& \quad \leq C \sum_{k=0}^{\infty} \frac{1}{k !} \sum_{u, v}\left|a_{u v}^{\prime}\right|\left|\xi^{u} \eta^{v}\right| \sum_{|\gamma|=m}\left\|D^{\gamma} A\right\|_{\mathrm{BMO}}\left(\int_{|y-h|<2}|f(y)|^{p} d x\right)^{1 / p} \\
& \leq C \sum_{k=0}^{\infty} \frac{1}{k !}\left(\sum_{\alpha, \beta}\left|a_{\alpha, \beta}\right|\left|\xi^{\alpha} \eta^{\beta}\right|\right)^{k} \sum_{|\gamma|=m}\left\|D^{\gamma} A\right\|_{\mathrm{BMO}}\left(\int_{|y-h|<2}|f(y)|^{p} d x\right)^{1 / p}
\end{aligned}
$$

where $\eta=(2,2, \ldots, 2)$. Note that

$$
\sum_{k=0}^{\infty} \frac{1}{k !}\left(\sum_{\alpha, \beta}\left|a_{\alpha \beta}\right|\left|\xi^{\alpha} \eta^{\beta}\right|\right)^{k}=\exp \left(\sum_{\alpha, \beta}\left|a_{\alpha, \beta}\right|\left|\xi^{\alpha} \eta^{\beta}\right|\right) \leq C
$$

we get

$$
\left(\int_{|x-h|<1}\left|S^{A} f(x)\right|^{p} d x\right)^{1 / p} \leq C \sum_{|\gamma|=m}\left\|D^{\gamma} A\right\|_{\mathrm{BMO}}\left(\int_{|y-h|<2}|f(y)|^{p} d y\right)^{1 / p} .
$$


Therefore, integrating the last inequality with respect to $h$, we obtain

$$
\left\|S^{A} f\right\|_{p} \leq C \sum_{|\gamma|=m}\left\|D^{\gamma} A\right\|_{\mathrm{BMO}}\|f\|_{p},
$$

which is (ii).

(ii) implies (i): Let $k$ and $l$ be two positive integers and $P(x, y)$ a non-degenerate realvalued polynomial with degree $k$ in $x$ and $l$ in $y$. Write

$$
P(x, y)=\sum_{|\alpha| \leq k,|\beta| \leq l} a_{\alpha \beta} x^{\alpha} y^{\beta} .
$$

By the dilation invariance, we may assume that $\sum_{|\alpha|=m,|\beta|=l}\left|a_{\alpha \beta}\right|=1$. Split $T^{A}=T_{0}^{A}+T_{\infty}^{A}$ as before. For $T_{\infty}^{A}$, since $P(x, y)$ is non-trivial, by the methods similar to those in the proof of (3.7), we can prove that

$$
\left\|T_{\infty}^{A} f\right\|_{p} \leq C \sum_{|\gamma|=m}\left\|D^{\gamma} A\right\|_{\mathrm{BMO}}\|f\|_{p} .
$$

In what follows, we shall prove that

$$
\left\|T_{0}^{A} f\right\|_{p} \leq C(n, \operatorname{deg} P) \sum_{|\gamma|=m}\left\|D^{\gamma} A\right\|_{\mathrm{BMO}}\|f\|_{p} .
$$

Let us begin with a double induction on the degrees in $x$ and $y$ of the polynomial. If $P(x, y)$ depends only on $x$ or only on $y$, it is obvious that the condition (ii) implies (4.2). We assume that (4.2) holds for all polynomials which are sum of monomials of degree less than $k$ in $x$ times monomials of any degree in $y$, together with monomials which are of degree $k$ in $x$ times monomials which are of degree less than $l$ in $y$. Rewrite

$$
P(x, y)=\sum_{|\alpha|=k,|\beta|=l} a_{\alpha \beta}\left(x^{\alpha} y^{\beta}-y^{\alpha+\beta}\right)+P_{0}(x, y),
$$

where $P_{0}(x, y)$ satisfies the inductive assumption. We split $T_{0}^{A}$ into

$$
\begin{aligned}
T_{0}^{A} f(x)= & \int_{|x-y|<1} e^{i P_{0}(x, y)} \frac{\Omega(x-y)}{|x-y|^{n+m}} R_{m+1}(A ; x, y) f(y) d y \\
& +\int_{|x-y|<1}\left(e^{i P(x, y)}-e^{i P_{0}(x, y)}\right) \frac{\Omega(x-y)}{|x-y|^{n+m}} R_{m+1}(A ; x, y) f(y) d y \\
:= & T_{0,1}^{A} f(x)+T_{0,2}^{A} f(x) .
\end{aligned}
$$

Now by our induction assumption we have

$$
\left\|T_{0,1}^{A} f\right\|_{p} \leq C \sum_{|\gamma|=m}\left\|D^{\gamma} A\right\|_{\mathrm{BMO}}\|f\|_{p} .
$$

On the other hand, if $|x|<1$ and $|x-y| \leq 1$, then it is easy to see that

$$
\left|e^{i P(x, y)}-e^{i P_{0}(x, y)}\right| \leq C \sum_{|\alpha|=k,|\beta|=l}\left|a_{\alpha \beta}\right||x-y|=C|x-y| .
$$


Denote $f_{0}(y)=f(y) \chi_{\{|y| \leq 2\}}(y)$. Then $T_{0,2}^{A} f(x)=T_{0,2}^{A} f_{0}(x)$, if $|x|<1$. Thus,

$$
\begin{aligned}
\left|T_{0,2}^{A} f(x)\right| & \leq C \int_{|x-y|<1}\left|\frac{\Omega(x-y)}{|x-y|^{n+m-1}} R_{m+1}(A ; x, y) f_{0}(y)\right| d y \\
& \leq C M_{\Omega}^{A} f_{0}(x) .
\end{aligned}
$$

By Lemma 2, we have

$$
\int_{|x|<1}\left|T_{0,2}^{A} f(x)\right|^{p} d x \leq C\left(\sum_{|\gamma|=m}\left\|D^{\gamma} A\right\|_{\mathrm{BMO}}\right)^{p} \int_{|y|<2}|f(y)|^{p} d y .
$$

Using the same argument as that used in [19, p. 189], we can get that

$$
\int_{|x-h|<1}\left|T_{0,2}^{A} f(x)\right|^{p} d x \leq C\left(\sum_{|\gamma|=m}\left\|D^{\gamma} A\right\|_{\mathrm{BMO}}\right)^{p} \int_{|y-h|<2}|f(y)|^{p} d y .
$$

Integrating the above inequality with respect to $h$, we obtain

$$
\left\|T_{0,2}^{A} f\right\|_{p} \leq C \sum_{|\gamma|=m}\left\|D^{\gamma} A\right\|_{\mathrm{BMO}}\|f\|_{p} .
$$

Therefore, (4.2) follows from (4.3) and (4.4). This finishes the proof of Theorem 1.

5. Further Results. Consider the multilinear oscillatory singular integral operator defined by

$$
T^{A_{1}, \ldots, A_{k}} f(x)=\text { p.v. } \int_{\boldsymbol{R}^{n}} e^{i P(x, y)} \frac{\Omega(x-y)}{|x-y|^{n+M}} \prod_{j=1}^{k} R_{m_{j}+1}\left(A_{j} ; x, y\right) f(y) d y,
$$

where $n \geq 2, k \geq 2, m_{j} \geq 1, j=1,2, \ldots, k, M=\sum_{j=1}^{k} m_{j}$ and $D^{\alpha_{j}} A_{j} \in$ BMO for $\left|\alpha_{j}\right|=$ $m_{j}, \quad j=1,2, \ldots, k$. For $\Omega \in B_{q}^{0, k}\left(S^{n-1}\right)$, repeating the arguments of the theorems, we can obtain the same conclusions as the theorems with bounds $\prod_{j=1}^{k} \sum_{\gamma_{j}=m_{j}}\left\|D^{\gamma_{j}} A_{j}\right\|_{\mathrm{BMO}}$. Here we omit the details.

In addition, if some restrictive conditions are imposed on the BMO functions $A_{j}$, we can obtain some more delicate results. First, we review a notion as follows.

DEFINITION 4 (cf. [6]). A local integrable function $a(x)$ will be said to belong to $\operatorname{BLO}\left(\boldsymbol{R}^{n}\right)$ if there is a constant $C$ such that for any cube $Q$

$$
m_{Q}(a)-\inf _{x \in Q} a(x) \leq C,
$$

where $m_{Q}(a)=|Q|^{-1} \int_{Q} a(x) d x$.

If $a \in \operatorname{BLO}\left(\boldsymbol{R}^{n}\right)$, then we denote $\|a\|_{\mathrm{BLO}\left(\boldsymbol{R}^{n}\right)}=\sup _{Q}\left\{m_{Q}(a)-\inf _{x \in Q} a(x)\right\}$.

Obviously, $L^{\infty}\left(\boldsymbol{R}^{n}\right) \subset \operatorname{BLO}\left(\boldsymbol{R}^{n}\right) \subset \operatorname{BMO}\left(\boldsymbol{R}^{n}\right)$ and if $a \in \operatorname{BLO}\left(\boldsymbol{R}^{n}\right)$, then

$$
\|a\|_{\mathrm{BMO}\left(\boldsymbol{R}^{n}\right)} \leq 2\|a\|_{\mathrm{BLO}\left(\boldsymbol{R}^{n}\right)} .
$$

Similarly to Theorems 1 and 2, we have the following results. 
THEOREM 3. Suppose that $\Omega$ is homogeneous of degree zero on $\boldsymbol{R}^{n}, k \geq 1$, and $A_{j}$ has derivatives of order $m_{j}\left(m_{j} \geq 1\right)$ in $\operatorname{BMO}\left(\boldsymbol{R}^{n}\right), j=1,2, \ldots, k$. If $D^{\alpha_{j}} A_{j}(x) \in \operatorname{BLO}\left(\boldsymbol{R}^{n}\right)$ and $D^{\alpha_{j}} A_{j}(x)$ is subharmonic for $\left|\alpha_{j}\right|=m_{j}, j=1,2, \ldots, k, \Omega \in B_{q}^{0,0}\left(S^{n-1}\right)$ for $q>1$, then for the operator $T^{A_{1}, \ldots, A_{k}}$ defined by (5.1), the corresponding conclusions of Theorems 1 and 2 with bound $C \prod_{j=1}^{k} \sum_{\left|\gamma_{j}\right|=m_{j}}\left\|D^{\gamma_{j}} A_{j}\right\|_{\mathrm{BLO}}$ also hold.

REMARK 2. It is worth pointing out that a BMO function $a(x)$ satisfying the restrictive conditions in Theorem 3 exists. A typical example is $\log |x|$.

Note that for any cube $Q,\left(|Q|^{-1} \int_{Q}\left|a(x)-a_{Q}\right|^{t} d x\right)^{1 / t} \leq\|a\|_{\mathrm{BLO}}(t \geq 1)$ if $a \in$ $\operatorname{BLO}\left(\boldsymbol{R}^{n}\right)$ and $a(x)$ is subharmonic. Then the same arguments as those used in proving Theorems 1 and 2, we can prove Theorem 3. We omit the details.

\section{REFERENCES}

[1] A. J. AL-HaSAn AND D. FAn, A singular integral operator related to block spaces, Hokkaido Math. J. 28 (1999), 285-299.

[2] A. P. CAlderón And A. Zygmund, On singular integrals, Amer. J. Math. 78 (1956), 289-309.

[ 3 ] W. G. Chen, G. E. Hu AND S. Z. LU, A criterion of $\left(L^{p}, L^{r}\right)$ boundedness for a class of multilinear oscillatory singular integrals, Nagoya Math. J. 149 (1998), 33-51.

[4] Z. F. Chu, G. E. HU AND Z. B. LU, A note on the multilinear oscillatory singular integral operators, Hiroshima Math. J. 31 (2) (2001), 201-212.

[ 5 ] J. Cohen And J. Gosselin, A BMO estimate for multilinear singular integral, Illinois J. of Math. 30 (1986), 445-464.

[ 6 ] R. Coifman And R. Rochberg, Another characterization of BMO, Proc. Amer. Math. Soc. 79 (2) (1980), 249-254.

[ 7 ] Y. DING AND S. Z. LU, Weighted $L^{p}$-boundedness for higher order commutators of oscillatory singular integrals, Tôhoku Math. J. 48 (1996), 437-449.

[ 8 ] Y. DING, S. Z. LU AND D. C. YANG, A criterion on weighted $L^{p}$-boundedness for rough multilinear Oscillatory singular integral operators, Proc. Amer. Math. Soc. 129 (2000), 1127-1136.

[ 9 ] G. E. HU, Multilinear oscillatory singular integral with rough kernel, Adv. Math. (China) 26 (1997), 50-59.

[10] Y. S. JiAng AND S. Z. Lu, Oscillatory singular integrals with rough kernel, in Harmonic Analysis in China (M.Cheng et al., eds.), Kluwer Academic Publishers (1995), 135-145.

[11] F. John And L. Nirenberg, On functions of bounded mean oscillation, Comm. Pure Appl. Math. 14 (1961), 415-426.

[12] M. Keitoku And E. SAto, Block spaces on the unit sphere in $\boldsymbol{R}^{n}$, Proc. Amer. Math. Soc. 119 (1993), 453-455.

[13] R. LONG, The spaces generated by blocks, Scientia Sinica A XXI II (1984), 16-26.

[14] S. Z. LU, On block decomposition of functions, Scientia Sinica A XXVII (1984), 585-596.

[15] S. Z. Lu, M. H. TAibleson And G. Weiss, Spaces Generated by Blocks, Beijing Normal University Press, Beijing, 1989.

[16] S. Z. LU AND H. X. WU, Oscillatory singular integrals and commutators with rough kernels, Annales des Sciences Mathématiques du Québec 27 (2003), 47-66.

[17] S. Z. LU AND Y. ZHANG, Criterion on $L^{p}$-boundedness for a class of oscillatory singular integrals with rough kernels, Rev. Mat. Iberoamericana 8 (1992), 201-219.

[18] Y. Meyer, M. Taibleson and G. Weiss, Some functional analytic properties of the space $B_{q}$ generated by blocks, Indiana. Univ. Math. J. 34 (1985), 493-515. 
[19] F. Ricci And E. M. Stein, Harmonic analysis on nilpotent groups and singular integrals, I. Oscillatory integrals, J. Funct. Analysis 73 (1987), 179-194.

[20] F. SORIA, Class of functions generated by blocks and associated Hardy spaces, Ph. D. Thesis, Washington University, St. Louis, 1983.

[21] E. M. SEIN, Singular integral and differentiability properties of functions, Princeton Univ. Press, 1971.

[22] M. H. TAiblesOn AND G. Weiss, Certain function spaces associated with a.e. convergence of Fourier series, Vol. I, Univ. of Chicago Conf. in honor of Zygmund, Woodsworth, 1983.

DEPARTMENT OF MATHEMATICS BEIJING NORMAL UNIVERSITY BEIJING 100875

People's Republic of ChinA

E-mail address: lusz@bnu.edu.cn
School of Mathematical Sciences

XIAMEN UNIVERSITY

Xiamen Fujian 361005

PEOPLE'S REPUBLIC OF CHINA

E-mail address: huoxwu@xmu.edu.cn 